\title{
Between Atomism and Superatomism
}

\section{T. Scott Dixon ${ }^{1,2}$}

Received: 16 May 2019 / Accepted: 19 March 2020 / Published online: 29 April 2020

(C) The Author(s) 2020

\begin{abstract}
There are at least three vaguely atomistic principles that have come up in the literature, two explicitly and one implicitly. First, standard atomism is the claim that everything is composed of atoms, and is very often how atomism is characterized in the literature. Second, superatomism is the claim that parthood is well-founded, which implies that every proper parthood chain terminates, and has been discussed as a stronger alternative to standard atomism. Third, there is a principle that lies between these two theses in terms of its relative strength: strong atomism, the claim that every maximal proper parthood chain terminates. Although strong atomism is equivalent to superatomism in classical extensional mereology, it is strictly weaker than it in strictly weaker systems in which parthood is a partial order. And it is strictly stronger than standard atomism in classical extensional mereology and, given the axiom of choice, in such strictly weaker systems as well. Though strong atomism has not, to my knowledge, been explicitly identified, Shiver appears to have it in mind, though it is unclear whether he recognizes that it is not equivalent to standard atomism in each of the mereologies he considers. I prove these logical relationships which hold amongst these three atomistic principles, and argue that, whether one adopts classical extensional mereology or a system strictly weaker than it in which parthood is a partial order, standard atomism is a more defensible addition to one's mereology than either of the other two principles, and it should be regarded as the best formulation of the atomistic thesis.
\end{abstract}

Keywords Atomism · Parthood · Fusion · Composition · Gunk

Funding support for the research carried out in this article comes in part from the Alexander von Humboldt Foundation.

T. Scott Dixon

ts.dixon@ashoka.edu.in

1 Department of Philosophy, Ashoka University, Plot No. 2 Rajiv Gandhi Education City, Rai, 131029, Sonipat, Haryana, India

2 Present address: Institute of Philosophy, Universität Hamburg, Überseering 35 \#4, 3. OG, R. 03089, 22297 Hamburg, Germany 


\section{Introduction}

There are at least three vaguely atomistic principles that have come up in the literature, two explicitly and one implicitly. Standard atomism is the claim that everything is composed of atoms. Superatomism is the claim that parthood is well-founded, which implies that every proper parthood chain terminates. And strong atomism is the claim that every maximal proper parthood chain terminates. I will introduce the reader to these principles more carefully at the end of this section. For now, I want to focus on the background of my project and its aims. Standard atomism is very often how atomism is characterized in the literature. ${ }^{1}$ Superatomism has been discussed as a stronger alternative to standard atomism [3, 21]. Strong atomism lies between these two theses in terms of its relative strength. Although strong atomism is equivalent to superatomism in classical extensional mereology, it is strictly weaker than it in strictly weaker systems in which parthood is a partial order. And it is strictly stronger than standard atomism in classical extensional mereology and, given the axiom of choice, in such strictly weaker systems as well. Though strong atomism has not, to my knowledge, been explicitly identified, Shiver [17] appears to have it in mind, though it is unclear whether he recognizes that it is not equivalent to standard atomism in each of the mereologies he considers.

In Sections 2 and 3, I prove the logical relationships which hold amongst these three atomistic principles in non-classical extensional mereology and some common strictly weaker mereologies in which parthood is a partial order. In Section 4, I prove the ones which hold in classical extensional mereology. In Section 5, I argue that, whether one adopts classical extensional mereology or a strictly weaker mereology in which parthood is a partial order, standard atomism is a more defensible addition to one's ontology than either of the other two principles, and it should be regarded as the best formulation of the atomistic thesis. I conclude in Section 6 by briefly summarizing my results and their implications. First, however, I must outline the different mereologies to which I intend my results to apply. Second, I must properly introduce standard atomism, strong atomism, and superatomism. These tasks constitute the remainder of this section.

I follow common practice, and take the relation expressed by 'is part of' as primitive, and define other related notions (such as composition) in terms of it. I abbreviate $\ulcorner x$ is part of $y\urcorner$ with $\ulcorner x \unlhd y\urcorner$. Most (though not all) hold that parthood is at least a partial order, and so is reflexive, antisymmetric, and transitive.

Reflexivity of Parthood. For any $x, x \unlhd x$.

Antisymmetry of Parthood. For any $x$ and $y$, if $x \unlhd y$ and $y \unlhd x$, then $x=y$.

Transitivity of Parthood. For any $x, y$, and $z$, if $x \unlhd y$ and $y \unlhd z$, then $x \unlhd z$.

Core mereology $(\mathbf{M})$ is the mereology which consists of just these axioms. ${ }^{2}$ While some of my proofs below presuppose less than that parthood is a partial order, others presuppose at least that much. It is for this reason that I consider mereologies only as

\footnotetext{
${ }^{1}$ For example, atomism is explicitly characterized in this way by Simons [18, 41], Uzquiano [21], and Varzi [24].

${ }^{2}$ I will largely, but not exclusively, follow the terminology in [23].
} 
weak as $\mathbf{M}$. Henceforth, I refer to mereologies in which the axioms of $\mathbf{M}$ are all true as 'M-mereologies'.

Many think that there is more to parthood than that it is a partial order. To help explicate these stronger mereological systems, and because they will be helpful in general in what follows, I define two other mereological notions. Let $\ulcorner x \triangleleft y\urcorner$ abbreviate $\ulcorner x$ is a proper part of $y\urcorner$ and let $\ulcorner x \circ y\urcorner$ abbreviate $\ulcorner x$ overlaps $y\urcorner$.

Proper Parthood. $x \triangleleft y=d f x \unlhd y$ and $x \neq y$.

Overlap. $x \circ y=d f x$ and $y$ share a part.

Certain principles which we would expect to govern these notions can be derived given the above axiomatization of parthood. Of particular relevance will be the result that proper parthood is a strict partial order, i.e., it is irreflexive and transitive (and so also asymmetric).

Irreflexivity of Proper Parthood. For every $x, x \nless x$.

Transitivity of Proper Parthood. For any $x, y$, and $z$, if $x \triangleleft y$ and $y \triangleleft z$, then $x \triangleleft z$.

Asymmetry of Proper Parthood. For any $x$ and $y$, if $x \triangleleft y$ then $y \not x$.

The most common mereological systems stronger than $\mathbf{M}$ include minimal mereology (MM), which adds weak supplementation to $\mathbf{M}$.

Weak Supplementation. For any $x$ and $y$, if $y \triangleleft x$, then some part of $x$ does not overlap $y$.

They also include extensional mereology (EM), which adds strong supplementation to $\mathbf{M}$.

Strong Supplementation. For any $x$ and $y$, if $x \not y$, then some part of $x$ does not overlap $y$.

Strong supplementation implies weak supplementation, given the antisymmetry of parthood. And MM allows for models that EM doesn't. (See [23, Section 3.2]). Thus MM is strictly weaker than EM when it is assumed that parthood is a partial order.

The strongest system I will consider is classical extensional mereology (CEM), which is a strengthening of EM. To explicate it, I must introduce the idea of a mereological fusion or sum. Roughly, a thing $x$ fuses (or is a sum of) some things when those things make up $x$ in its entirety, with each contributing, in a mereological sense of 'make up'. There is more to me than just my legs, and so I do not fuse them. I do, however, fuse my legs, torso, arms, and head. My coffee cup, on the other hand, plays no role in making me up. So it is not even among any things which I fuse. The notion of fusion has been formally defined in different ways. There are three that are standard (see, for example, [8] and [23, Section 4.3] and [24, 2893-94]). In what follows, ' $\Gamma$ ' and ' $\Delta$ ' are plural variables and $\ulcorner\Gamma \preceq \Delta\urcorner$ abbreviates $\ulcorner\Gamma$ are among $\Delta\urcorner{ }^{3}$

\footnotetext{
${ }^{3}$ Many authors define these notions in first-order logic with only singular quantification. I employ plural quantification, since some of the atomistic principles which interest me cannot be stated using singular first-order logic. See fn. 7 below for more on this issue. I adopt as my underlying language a logic like Oliver and Smiley's [15] full plural logic, though I certainly do not make use of all of its expressive power.
} 
Fusion 1. $x$ fuses $1 \Gamma={ }_{d f}$ (i) every $y \preceq \Gamma$ is part of $x$ and (ii) every part of $x$ overlaps at least one $y \preceq \Gamma$.

Fusion 2. $x$ fuses $_{2} \Gamma={ }_{d f}$ (i) $x$ overlaps all and only those things that overlap at least one $y \preceq \Gamma$.

Fusion 3. $x$ fuses $_{3} \Gamma=_{d f}$ (i) every $y \preceq \Gamma$ is part of $x$ and (ii) $x$ is part of all those things whose parts include every $y \preceq \Gamma$.

CEM results from adding to EM the claim that any things have a fusion of the first or second type, i.e., where $n \in\{1,2\}$,

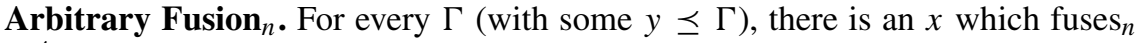
$\Gamma{ }^{4}$

In CEM, the above three definitions of fusion are equivalent (see [8, 81]), and so in such a context, one may speak of something that fuses some things without specifying which sense of fusion one has in mind. This completes my outline of the mereologies to which I intend my results to apply. They consist of M, MM, EM, and CEM.

I turn now to a more thorough introduction to the three vaguely atomistic principles with which I am concerned.

Standard Atomism. Everything is composed of atoms.

Superatomism. Parthood is well-founded.

Strong Atomism. Every maximal proper parthood chain terminates.

The first principle involves the notion of composition, which is characterized as nonoverlapping fusion. Fusion and composition share the feature that the things that are fused by or that compose something make it up in its entirety, with each contributing. The difference between them consists in the fact that the former allows for overlap amongst the things, while the latter does not. So, while I fuse my top two thirds and bottom two thirds, I am not composed of those two things. However, I both fuse and am composed of my top half and bottom half. Since there are three definitions of fusion (which are not always equivalent in systems weaker than CEM), there are three definitions of composition, each of which is an instance of the following schema, where $n \in\{1,2,3\}$.

Composition $_{n} \cdot x$ is composed $_{n}$ of $\Gamma={ }_{d f}$ (i) $x$ fuses $_{n} \Gamma$ and (ii) $\Gamma$ are pairwise disjoint (i.e., no two overlap). ${ }^{5}$

The latter two atomistic principles can be unpacked with the help of the following definitions.

\footnotetext{
${ }^{4}$ Since I use a plural first-order language, I can state this without invoking specifiable sets, as is standard practice in developments of mereology in singular first-order logic. The axiomatization of CEM using arbitrary fusion $_{1}$ contains redundancy, since CEM results from just it along with the transitivity of parthood and weak supplementation (see [8, 66-67]). Adding arbitrary fusion 3 to EM results in a system slightly weaker than CEM (see [23, Section 4.4]), which yields CEM once another axiom, such as filtration (i.e., the claim that if something is part of a fusion 3 of some things then it overlaps at least one of those things), is added (see [8, 67-68]).

${ }^{5}$ See $[24,2893]$. Van Inwagen [22, 29] notably makes use of the clause that $\Gamma$ are pairwise disjoint to distinguish composition claims from mere fusion claims (though he endorses fusion 1).
} 
Well-Foundedness of Parthood. $\unlhd$ is well-founded $={ }_{d f}$ every non-empty subset $\mathrm{S}$ of the domain of $\unlhd$ has a $\unlhd$-minimal element, i.e., an element $y$ such that, for every $x \in \mathrm{S}, x \notin y$.

(Proper Parthood) Chains. $\Gamma$ form a (proper parthood) chain $=_{d f}$ (i) there are $x, y \preceq \Gamma$ such that $x \triangleleft y$ and (ii) for every $x, y \preceq \Gamma$, either $x \triangleleft y, y \triangleleft x$, or $x=y$. (Downwardly) Maximal Chains. $\Gamma$ form a (downwardly) maximal chain $=_{d f}$ (i) $\Gamma$ form a chain and (ii) there is no $y$ that is a proper part of every $x \preceq \Gamma$.

(Downwardly) Terminating Chains. $\Gamma$ form a (downwardly) terminating chain $=_{d f}$ (i) $\Gamma$ form a chain and (ii) there is a $y \preceq \Gamma$ that is a proper part of every other $x \preceq \Gamma$.

A couple of things are worth noting about these three principles. First, the claim that parthood is well-founded is equivalent to the claim that every proper parthood chain terminates, given the principle of dependent choices. The left-to-right direction of the equivalence is provable without this principle, though it requires the assumption that every chain is set-sized. Such a proof appears below in my proof of Proposition $2 .^{6}$ Second, while standard atomism can be stated in singular first-order logic, neither strong atomism nor superatomism can be. ${ }^{7}$

\footnotetext{
${ }^{6}$ I omit a proof of the right-to-left direction, since I do not rely on it in what follows. It is provable given the relevant instance of the principle of dependent choices: if, for any $x$ in some non-empty set, there is a $y$ in that set such that $y \triangleleft x$ (something which holds of any non-terminating chain), then there is a chain $x_{0}, x_{1}, \ldots, x_{n}, \ldots$ in that set such that $x_{n+1} \triangleleft x_{n}$ for every $n \in\{0,1, \ldots\}$. While weaker than the axiom of choice, the principle of dependent choices is stronger than the countable axiom of choice $[9,50]$.

${ }^{7}$ This can be shown by the same method Boolos [2, 432-33] uses to show that the Geach-Kaplan sentence ('Some critics admire only one another') is, as he puts it, 'nonfirstorderizable'. Boolos's method depends on the observation that every consistent (singular) first-order theory of arithmetic has both standard and non-standard models [11]: $\mathrm{n}$. 2. Consider (what I will show to be) the weaker of the two principles: strong atomism. An arithmetical interpretation of it is true in the standard model of arithmetic but false in every non-standard model. To construct one such interpretation, let $\sqsubset$ be the transitive closure of the predecessor relation on a given set $\mathrm{S}$, i.e., let $\sqsubset$ be the smallest relation on $\mathrm{S}$ such that, for any $x, y$, and $z$ in $\mathrm{S}$, if $x$ is a predecessor of $y$, then $x \sqsubset y$ and (ii) if $x \sqsubset y$ and $y \sqsubset z$ then $x \sqsubset z$. Then interpret ' $\triangleleft$ ' as $\sqsubset$ and let S be the domain of any given model. The resulting arithmetical interpretation of strong atomism says that every $\sqsubset$-maximal $\sqsubset$-chain terminates. This is true in the standard model, since every such chain terminates in 0 . But it is false in every non-standard model. Every non-standard element in every non-standard model has a predecessor. But none of the standard elements are predecessors of any non-standard element. This means that every non-standard model contains a non-terminating $\sqsubset$-chain of non-standard elements. (Here I am relying on the principle of dependent choices — see fn. 6.) Moreover, every such chain is $\sqsubset$-maximal. Consider such a chain C. There is no $y$ such that $y \sqsubset x$ for every $x \in \mathrm{C}$. If there were, the definition of $\sqsubset$ would guarantee that $y$ is a predecessor of some $x \in \mathrm{C}$. But since the predecessor of any element of any model of arithmetic (standard or non-standard) is unique, $y$ must be in C. But then $y \sqsubset y$, which would mean that $y<y$, which is false of every element of every model of arithmetic. A similar case can be made for the nonfirstorderizability of superatomism by interpreting ' $\triangleleft$ ' as $<$, though, since I show below that superatomism is stronger than strong atomism (given the axiom of choice), there is no need to present this case in detail. Many thanks to Michael Glanzberg (personal communication) for a helpful discussion about nonfirstorderizability. Uzquiano [21, n. 11] provides a first-order schematization of superatomism, but the resulting instances apply only to subsets of the domain that are definable in singular first-order logic, which are outnumbered by subsets of the domain whenever the domain is infinite. So the schematization is not as strong as his second-order or my plurally quantified formulation. Strong atomism can be schematized as well. Let every instance of the following schema be true:
}

if $\{x: \varphi(x)\}$ is a maximal chain, then $\{x: \varphi(x)\}$ terminates, 


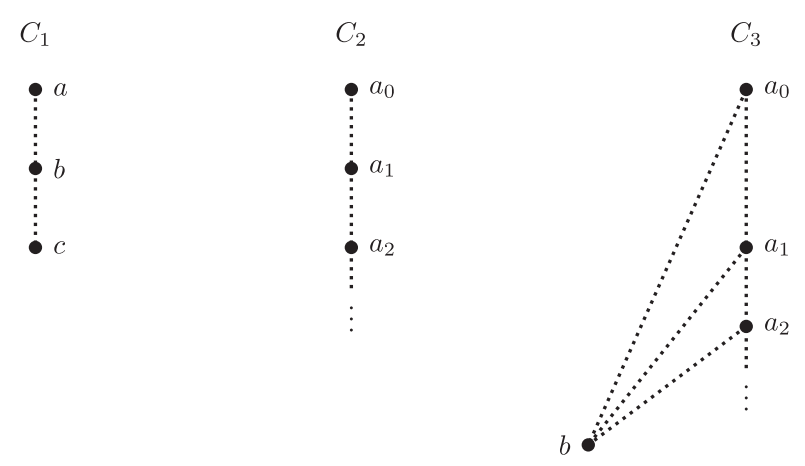

Fig. 1 An assortment of proper parthood chains. A dotted line running in a downward direction from a node $x$ to another node $y$ (which may run through one or more other nodes) indicates that $y$ is a proper part of $x$.

To get an idea of the differences between terminating and non-terminating chains, and between maximal and non-maximal chains, consult Fig. 1. It depicts three chains: one which contains no non-terminating chains, i.e., parthood is well-founded on it, and two which contain such chains. $\mathrm{C}_{1}$ terminates, since something $(c)$ is a proper part of everything else in it. Its subchains include itself, the chain formed by $a, b$, and the chain formed by $b, c$. It is maximal, since (as depicted) nothing is a proper part of everything in it. The chain formed by $a, b$ is not maximal, since $c$ is a proper part of everything in it. But the chain formed by $b, c$ is maximal, since, like $\mathrm{C}_{1}$ itself, nothing is a proper part of everything in it. $\mathrm{C}_{2}$ does not terminate, since nothing in it is a proper part of everything else in it. It contains an infinite number of subchains, including itself. It is maximal. Each subchain formed by $a_{n_{0}}, a_{n_{1}}, \ldots a_{n_{k}}$, where $n_{i} \in\{0,1, \ldots\}$ for every $i \in\{0, \ldots, k\}$ and, for every $i, j \in\{0, \ldots, k\}$, $n_{i}<n_{j}$ iff $i<j$, is not maximal, since there is something (e.g., $a_{n_{k}+1}$ ) that is a proper part of everything in it. But each subchain formed by $a_{n_{0}}, a_{n_{1}}, \ldots$, where $n_{i} \in\{0,1, \ldots\}$ for every $i \in\{0,1, \ldots\}$ and, for every $i, j \in\{0, \ldots, k\}, n_{i}<n_{j}$ iff $i<j$, is maximal, since, like $\mathrm{C}_{2}$ itself, nothing is a proper part of everything in it. $\mathrm{C}_{3}$ terminates, since something $(b)$ is a proper part of everything else in it. But it contains subchains which do not terminate, formed by $a_{n_{0}}, a_{n_{1}}, \ldots$, where $n_{i} \in\{0,1, \ldots\}$ for every $i \in\{0,1, \ldots\}$ and, for every $i, j \in\{0,1, \ldots\}, n_{i}<n_{j}$ iff $i<j . \mathrm{C}_{3}$ itself is maximal, as is every subchain of it terminating in $b$, since nothing is a proper part of everything in any of them. No subchain consisting of just $a$ s is maximal, since there is something (at least $b$ ) that is a proper part of everything in each of them.

One can start to get an idea of the differences amongst standard atomism, strong atomism, and superatomism by taking note of what each would say about each of the chains in Fig. 1 in any $\mathbf{M}$-mereology. Superatomism allows for $\mathrm{C}_{1}$ but neither $\mathrm{C}_{2}$ nor $\mathrm{C}_{3}$. Strong atomism allows for $\mathrm{C}_{1}$ and $\mathrm{C}_{3}$ but not $\mathrm{C}_{2}$. Standard atomism would allow for all three chains on all three definitions of composition if otherwise

where $\varphi(\mathbf{u})$ is a first-order formula in which $\mathbf{u}$ is a free variable. But this schematization is also weaker than my plurally quantified formulation. 
inconsequential additions are made to them, such as adding an immediate atomic proper part to every composite object in each chain, where

Atomicness. $x$ is atomic $={ }_{d f} x$ has no proper parts.

Compositeness. $x$ is composite $=d f x$ has a proper part.

Immediate Proper Parthood. $x$ is an immediate proper part of $y={ }_{d f} x \triangleleft y$ and no $z$ both is a proper part of $y$ and has $x$ as a proper part (see $[14,415]$ ).

In what follows, it will emerge that the structures which result from these additions in the case of $\mathrm{C}_{2}$ and $\mathrm{C}_{3}$ are central to reasoning about the relative strengths of these atomistic principles.

\section{Superatomism and Strong Atomism in EM and Strictly Weaker M-Mereologies}

I begin the investigation with the logical relationships that hold amongst the three atomistic principles in EM and strictly weaker M-mereologies. In this section, I concern myself with the relationship which holds between superatomism and strong atomism. As I mentioned, the former is strictly stronger than the latter in such systems.

Proposition 1 Superatomism is strictly stronger than strong atomism in $\mathbf{E M}$ and strictly weaker M-mereologies.

I prove this by proving first that superatomism implies strong atomism in $\mathbf{M}$, and second that there is a model of EM in which strong atomism is true but superatomism is false. I begin with the implication claim.

Proposition 2 Superatomism implies strong atomism in $\boldsymbol{M}$.

My strategy is to prove first that superatomism implies that every chain terminates. The goal follows immediately.

Proof Suppose that every non-empty set $\mathrm{S}$ has a $\unlhd$-minimal element and consider an arbitrary chain formed by some objects $\mathrm{C}$. By the initial assumption, something, $m$, among $\mathrm{C}$ is such that, for every $x \preceq \mathrm{C}, x \not m$. Consider an arbitrary $a \neq m$ among C. Since $\mathrm{C}$ form a chain, such an $a$ exists. For the same reason, either $a \triangleleft m$, or $m \triangleleft a$. Since $m$ is $\unlhd$-minimal, we know that $a \not m$. So $m \triangleleft a$. And since $a$ is arbitrary, $m \triangleleft x$ for every $x$ among C. By the definition of terminating chains, the chain formed by $\mathrm{C}$ terminates. And since that chain is arbitrary, every chain terminates. This of course means that every maximal chain terminates.

This proof requires nothing of parthood except that it allows for proper parthood chains (and, because ' $\mathrm{C}$ ' denotes a non-trivial plurality - i.e., a plurality of two or more objects - that at least two objects exist, and that those chains are set-sized). The way I have defined chains, maximal chains, and terminating chains presupposes 
the transitivity of proper parthood which, as I show below, presupposes (only) the antisymmetry and transitivity of parthood. But they could be defined instead in terms of the transitive closure of proper parthood.

I turn now to the second component of Proposition 1.

Proposition 3 There is a model of $\boldsymbol{E M}$ in which strong atomism is true but superatomism is false.

When constructing models, I am careful to ensure that they qualify as models of the strongest of the systems in which I am interested (EM in this section and the next, CEM in the one following them) in order to ensure that the proponent of that system will countenance them. But it is important to note that they qualify as models of any strictly weaker system as well, since anything that is a model of a given mereology is a model of any strictly weaker one. This, along with the fact that my proofs of implication presuppose of parthood only that it is a partial order, ensures that my proofs of the relative strengths of the atomistic principles hold in as wide a range of mereologies as possible: from $\mathbf{M}$ to $\mathbf{E M}$ in this section and the next and to CEM in the one following them.

To see that Proposition 3 is true, define the model $\mathcal{M}_{1}$ as follows.

$\mathcal{M}_{1}=\left\langle\mathcal{D}_{1}, \triangleleft\right\rangle$, where $\mathcal{D}_{1}=\left\{a_{0}, a_{1}, \ldots, b, c_{0}, c_{1}, \ldots\right\}$ and the extension of $\triangleleft$ is given by:

(i) $a_{n} \triangleleft a_{m}$ iff $n>m$ for any $n, m \in\{0,1, \ldots\}$,

(ii) $b \triangleleft a_{n}$ for every $n \in\{0,1, \ldots\}$,

and

(iii) $c_{n} \triangleleft a_{m}$ iff $n \geq m$ for any $n, m \in\{0,1, \ldots\}$. $^{8}$

$\mathcal{M}_{1}$ is depicted in Fig. 2. $\mathcal{M}_{1}$ is a model of EM because (a) parthood is a partial order on its domain and (b) it satisfies strong supplementation, i.e, for any $x, y \in \mathcal{D}_{1}$, if $x \not y$, then some part of $x$ does not overlap $y$. There are six types of case to consider. Let $n, m \in\{0,1, \ldots\}$.

(i) $a_{n} \unlhd a_{m}$ if $n<m$, but $a_{n}$ has a part, e.g., $c_{n}$, which does not overlap $a_{m}$.

(ii) $a_{n} \unlhd b$ for any $n$, but $a_{n}$ has a part, e.g., $c_{m}$ for any $m \geq n$, which does not overlap $b$.

(iii) $\quad a_{n} \not c_{m}$ for any $n$ and $m$, but $a_{n}$ has a part, e.g., $b$, which does not overlap $c_{m}$.

(iv) $b \unlhd c_{n}$ for any $n$, but $b$ has a part, viz., itself, which does not overlap $c_{n}$.

(v) $c_{n} \unlhd a_{m}$ if $n<m$, but $c_{n}$ has a part, viz., itself, which does not overlap $a_{m}$.

(vi) $c_{n} \unlhd b$ for any $n$, but $c_{n}$ has a part, viz., itself, which does not overlap $b$.

Strong atomism is true in $\mathcal{M}_{1}$ because every maximal chain in $\mathcal{M}_{1}$ terminates. The maximal chains that exist in $\mathcal{M}_{1}$ are formed by

$$
a_{n}, c_{n} \text { for every } n \text {, }
$$

\footnotetext{
${ }^{8}$ For the sake of simplicity, I fix the extension of $\unlhd$ in this model and the one that follows by fixing the extension of $\triangleleft$. I leave it implicit that everything is part of itself.
} 
Fig. 2 Model $\mathcal{M}_{1}$

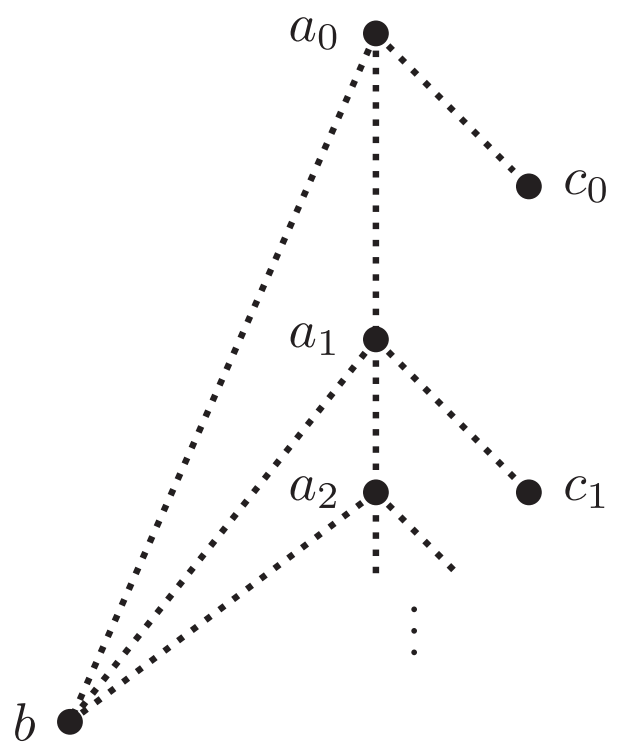

(ii) $a_{n}, a_{m_{0}}, a_{m_{1}}, \ldots, a_{m_{k}}, c_{m_{k}}$ for every $n$ and $m_{i}>n$ for every $i \in\{0, \ldots, k\}$, where, for any $i, j \in\{0, \ldots, k\}, m_{i}<m_{j}$ iff $i<j$,

(iii) $a_{n}, b$ for every $n$,

(iv) $a_{n}, a_{m_{0}}, a_{m_{1}}, \ldots, a_{m_{k}}, b$ for every $n$ and $m_{i}>n$ for every $i \in\{0, \ldots, k\}$, where, for any $i, j \in\{0, \ldots, k\}, m_{i}<m_{j}$ iff $i<j$

(v) $a_{n}, a_{m_{0}}, a_{m_{1}}, \ldots, b$ for every $n$ and $m_{i}>n$ for every $i \in\{0,1, \ldots\}$, where, for any $i, j \in\{0,1, \ldots\}, m_{i}<m_{j}$ iff $i<j$.

Each chain of each of these types terminates - in (i) $c_{n}$, (ii) $c_{m_{k}}$, (iii) $b$, and (iv) $b$, and (v) $b$ respectively. But superatomism is false in $\mathcal{M}_{1}$, since not all chains terminate. For example, the chain formed by $a_{n}, a_{n+1}, \ldots$ for every $n$ does not terminate.

\section{Strong Atomism and Standard Atomism in EM and Strictly Weaker M-Mereologies}

I turn now to the logical relationship which holds between strong atomism and standard atomism in EM and strictly weaker M-mereologies. As I mentioned, the former is strictly stronger than the latter in such systems given the axiom of choice.

Proposition 4 Strong atomism is strictly stronger than standard atomism in $\mathbf{E M}$ and strictly weaker M-mereologies given the axiom of choice.

I prove this by proving first that strong atomism implies standard atomism in $\mathbf{M}$ given the axiom of choice, and second that there is a model of $\mathbf{E M}$ in which standard atomism is true but strong atomism is false. I begin with the implication claim. 
Proposition 5 Strong atomism implies standard atomism in $\boldsymbol{M}$ given the axiom of choice.

I will not prove this claim directly. Instead, I will prove the following claim.

Proposition 6 Strong atomism implies atomicity in $\mathbf{M}$ given the axiom of choice.

Atomicity is the principle one typically adopts to ensure that all of the models of one's mereology are atomistic (see, for example, $[18,42]$ and [23, Section 3.4]).

Atomicity. Everything has an atomic part.

Varzi [24, Section 2] has proved that atomicity implies atomism.

Proposition 7 If everything has an atomic part then everything is composed of atoms (on all three of the standard definitions of fusion).

Two of Varzi's proofs - those involving the first two definitions of fusiondepend only on the assumption that parthood is reflexive and transitive, and are therefore sound in $\mathbf{M}$. That involving the third definition of fusion requires strong supplementation, and so holds only in extensional mereologies. But, as Varzi notes (Ibid.: 2899), non-extensional models are 'at odds with the idea that a sum is just a "minimal upper bound", which is what a fusion is according to the third definition. So this extra assumption does not impact the generality of his results (or, for the same reason, that of my own). Proposition 7 holds only assuming reflexivity and transitivity when fusion is interpreted by any of the two definitions which are consonant with M-mereologies strictly weaker than EM. That is the important thing.

My proof of Proposition 6 relies on no special assumptions about parthood except that it is a partial order. I invoke the transitivity of proper parthood, which can be proved using only the assumptions that parthood is antisymmetric and transitive. (I put uses of substantive assumptions - most importantly those about parthood - in boldface.)

Proof Consider arbitrary $a, b$, and $c$ and suppose that $a \triangleleft b$ and $b \triangleleft c$. By the definition of proper parthood, (i) $a \unlhd b$ and $a \neq b$ and (ii) $b \unlhd c$ and $b \neq c$. By the transitivity of parthood, $a \unlhd c$. Now suppose for reductio that $a=c$. Since $a \unlhd b$, $c \unlhd b$. And since $b \unlhd c$, the antisymmetry of parthood guarantees that $b=c$, contradicting the result that $b \neq c$. So $a \neq c$. By the definition of proper parthood, $a \triangleleft c$.

But my proof of Proposition 6 also relies on an instance of the Hausdorff maximal principle, which applies to partial orders. So, to be sure I can legitimately make use of it, I assume that parthood is a partial order and I define proper parthood as I have, which ensures that it is a partial order too. 
Hausdorff Maximal Principle. If $\triangleleft$ partially orders its domain, then for any $\triangleleft-$ chain formed by $\Gamma$, there are $\Delta$ such that (i) $\Delta$ form a maximal chain and (ii) $\Gamma \preceq \Delta .^{9}$

This principle is as strong as the axiom of choice. It allows one to choose a maximal chain from any partially ordered set, importantly, including any that is such that, for everything $x$ in it, there is something $y$ in it such that $y$ is less than $x$ in the partial order. ${ }^{10}$

The proof of Proposition 6 is rather involved. So I will simply provide an outline of it here. One can consult the Appendix for the complete proof. For both the proof and the outline, it is helpful to have in hand the notion of a final segment of a chain.

Definition of Final Segments. $\Gamma$ form a final segment of $\Delta={ }_{d f}$ (i) $\Delta$ form a chain, (ii) there is an $x \preceq \Gamma$, (iii) $\Gamma \preceq \Delta$, and (iv) every $x \preceq \Gamma$ is a proper part of every $y$ among $\Gamma$ but not among $\Delta$.

The chain formed by $b, c$ in Fig. 1, for example, is a final segment of $\mathrm{C}_{1}$. The chain formed by $a_{n}, a_{n+1}, \ldots$ for any $n \in\{0,1, \ldots\}$ is a final segment of $\mathrm{C}_{2}$. And the chain formed by $a_{n}, a_{n+1}, \ldots, b$ for any $n \in\{0,1, \ldots\}$ is a final segment of $\mathrm{C}_{3}$. Every chain is a final segment of itself. To prove Proposition 6, I begin by supposing that strong atomism is true and I consider an arbitrary $a$. I then set out to show that $a$ has an atomic part. I do this by reductio. That is, I assume that $a$ does not have an atomic part. I then focus on the collection $\mathrm{Z}$ of all and only $a$ 's proper parts. I establish several things about $Z$ to find a contradiction. First, I show that, for every $x \preceq Z$, there is a $y \preceq Z$ that is a proper part of $x$. Second, I apply Hausdorff's maximal principle, which allows me to choose a maximal chain, formed by some objects M, from Z. ${ }^{11}$ Third, I show that this chain has as a final segment a chain, formed by some objects $\mathrm{N}$, such that

(i) $\mathrm{N} \preceq \mathrm{Z}$,

(ii) the chain formed by $\mathrm{N}$ is maximal, and

(iii) the chain formed by $\mathrm{N}$ does not terminate. ${ }^{12}$

\footnotetext{
${ }^{9}$ The Hausdorff maximal principle is usually stated set-theoretically. I have given its counterpart in plural first-order logic.

${ }^{10}$ See $[4,56-63]$ for a proof of the equivalence of the Hausdorff maximal principle and the axiom of choice and several variants of it. For an earlier proof of the Hausdorff maximal principle using the well-ordering theorem, see [7, 197-98]. For one using the axiom of choice, see [6].

${ }^{11}$ I am unsure whether assuming something as strong as the Hausdorff maximal principle is required, though I suspect that it is. I do not see how to define a choice function that will enable one to choose a maximal chain from Z. And the axiom of countable choice (see, for example, $[9,50]$ ) isn't strong enough when the cardinality of the chain is uncountable. Nor does the principle of dependent choices (Ibid.) guarantee that the chain it selects is maximal. I am also unsure about the significance of this assumption. Any pretensions to consistency with the spirit of constructive mathematics is compromised in any case by my use of reductio in the proof. So the use of the principle doesn't make non-constructive what would otherwise be a constructive proof.

${ }^{12}$ It cannot be proven that $\mathrm{M} \preceq \mathrm{Z}$ because $a$ might itself be a proper part of something, in which case something would be among $\mathrm{M}$ that is not among $\mathrm{Z}$. When one applies the Hausdorff maximal principle to an appropriate set, one chooses a chain that is maximal in both directions, not just in the downward direction.
} 
Since the initial assumption that every maximal chain terminates ensures that the chain formed by $\mathrm{N}$ terminates, there is a contradiction.

I turn now to the second component of Proposition 4.

Proposition 8 There is a model of $\mathbf{E M}$ in which standard atomism is true but strong atomism is false.

Define the model $\mathcal{M}_{2}$ as follows.

$\mathcal{M}_{2}=\left\langle\mathcal{D}_{2}, \triangleleft\right\rangle$, where $\mathcal{D}_{2}=\left\{a_{0}, a_{1}, \ldots, b_{0}, b_{1}, \ldots\right\}$ and the extension of $\triangleleft$ is given by:

(i) $a_{n} \triangleleft a_{m}$ iff $n>m$ for any $n, m \in\{0,1, \ldots\}$

and

(ii) $b_{n} \triangleleft a_{m}$ iff $n \geq m$ for any $n, m \in\{0,1, \ldots\}$.

$\mathcal{M}_{2}$ is depicted in Fig. 3. $\mathcal{M}_{2}$ is a model of EM (and so of every strictly weaker Mmereology) because (a) parthood is a partial order on its domain and (b) it satisfies strong supplementation, i.e., for any $x, y \in \mathcal{D}_{2}$, if $x \unlhd y$, then some part of $x$ does not overlap $y$. There are two types of case to consider. Let $n, m \in\{0,1, \ldots\}$.

(i) $a_{n} \unlhd a_{m}$ if $n<m$, but $a_{n}$ has a part, e.g., $b_{n}$, which does not overlap $a_{m}$.

(ii) $\quad b_{n} \unlhd a_{m}$ if $n<m$, but $b_{n}$ has a part, viz., itself, which does not overlap $a_{m}$.

Standard atomism is true in $\mathcal{M}_{2}$, since $a_{n}$ is composed of $b_{n}, b_{n+1}, \ldots$ for every $n$. This follows given the extension of parthood in $\mathcal{M}_{2}$, no matter which of the three standard definitions of fusion one employs. For a proof of these claims, see [24, 2894], fn. 5. But strong atomism is false in $\mathcal{M}_{2}$, since not all maximal chains terminate. For example, the chain formed by $a_{n}, a_{n+1}, \ldots$ for every $n$ is maximal, but does not terminate.

$\mathcal{M}_{2}$ is isomorphic to a model discussed by both Shiver [17] and Varzi [24]. Shiver uses it in an attempt to show that atomicity is not an adequate characterization of atomism, since it allows for models with the structure of $\mathcal{M}_{2}$ when it shouldn't. But he seems to have in mind strong atomism, not standard atomism, as he rules the

Fig. 3 Model $\mathcal{M}_{2}$

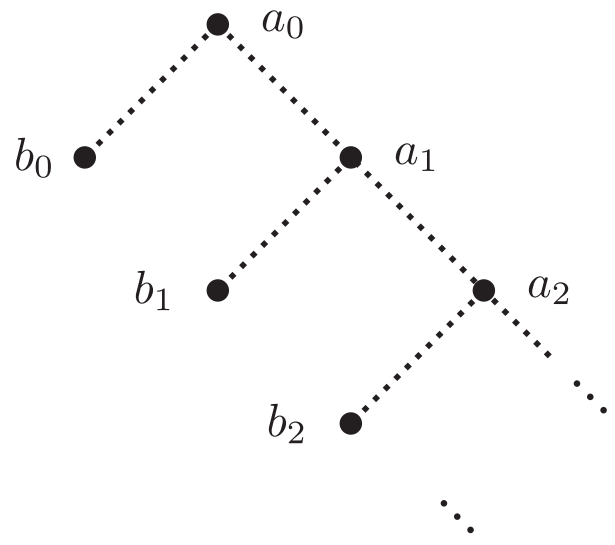


isomorph of $\mathcal{M}_{2}$ he discusses to be non-atomistic because it contains a maximal nonterminating chain and, he says, "if a poset has a non-terminating maximal chain, it follows that the poset is not atomistic" $[17,608]$. While this is certainly true of strong atomism, it is not true, as Varzi shows, of standard atomism. The two theses are not equivalent in EM and strictly weaker M-mereologies. Models with the structure of $\mathcal{M}_{2}$ show this. It might be worth adding that their mere non-equivalence does not depend upon the truth of the Hausdorff maximal principle.

I now sum up what I've done in this section and the last. I have shown that superatomism is strictly stronger than strong atomism in EM and strictly weaker mereologies, and that strong atomism is strictly stronger than standard atomism in such systems, given the Hausdorff maximal principle. I will discuss philosophical matters related to the non-equivalence of these three principles in section 5. Before that, I will investigate the logical relationships which hold amongst them in CEM, as they differ somewhat from the relationships which hold amongst them in the strictly weaker mereologies I have considered so far.

\section{Classical Extensional Mereology}

Things change when one assumes that any objects have a fusion of type 1 or type 2 . Neither $\mathcal{M}_{1}$ nor $\mathcal{M}_{2}$ is a model of CEM, and so they cannot be used to help establish the analogs of Propositions 1 and 4 for CEM. The problem with these two models is that it is not the case that, for any things $\Gamma$ in the domain of either, the domain contains something that fuses $\Gamma$. We can extend each of the models to include those fusions, and add in all of the resulting proper parthood claims. But in doing so we create new models. It turns out that a model of CEM with denumerably many atoms and in which standard atomism is true (as is the case in each of $\mathcal{M}_{1}$ and $\mathcal{M}_{2}$ ) can have only a single sort of structure. ${ }^{13}$ This is the structure isomorphic to that imposed by the subset relation on the set of all non-empty subsets of $\{0,1, \ldots\}$. When extended to ensure the truth of arbitrary fusion $n$ for $n \in\{1,2\}$, each of $\mathcal{M}_{1}$ and $\mathcal{M}_{2}$ results in a model with this singular structure. Define the model $\mathcal{M}_{3}$ as follows. Let $\mathcal{P}(\mathrm{S})$ be the powerset of any set $\mathrm{S}$.

$\mathcal{M}_{3}=\left\langle\mathcal{D}_{3}, \triangleleft\right\rangle$, where $\mathcal{D}_{3}=\mathcal{P}(\{0,1, \ldots\}) \varnothing$ and the extension of $\unlhd$ is given by:

- $\quad x \unlhd y$ iff $x \subseteq y$ for every $x, y \in \mathcal{D}_{3}$.

Due to its complexity, $\mathcal{M}_{3}$ cannot be depicted by a Hasse diagram. But fragments of it can. One such fragment is depicted in Fig. 4. $\mathcal{M}_{3}$ is a model of CEM because, in addition to the fact that parthood is a partial order in it, it satisfies strong supplementation and arbitrary fusions ${ }_{n}$ for $n \in\{1,2\}$. That $\mathcal{M}_{3}$ satisfies strong supplementation can be seen by noting that, for any sets $\mathrm{S}, \mathrm{T} \in \mathcal{D}_{3}$, if $\mathrm{S} \nsubseteq \mathrm{T}$, then some $\mathrm{U} \subseteq \mathrm{S}$ is such that $\mathrm{U} \cap \mathrm{T}=\varnothing$. And that it satisfies arbitrary fusions can be seen by noting that, for any sets $\mathrm{S}_{1}, \mathrm{~S}_{2}, \ldots \in \mathcal{D}_{3}$, there is a set $\mathrm{T} \in \mathcal{D}_{3}$ such that $T=\bigcup\left(\mathrm{S}_{1}, \mathrm{~S}_{2}, \ldots\right)$.

\footnotetext{
${ }^{13}$ Thanks to Cody Gilmore (personal communication) for bringing this to my attention, and to Paul Hovda (personal communication) for a helpful discussion about this issue.
} 


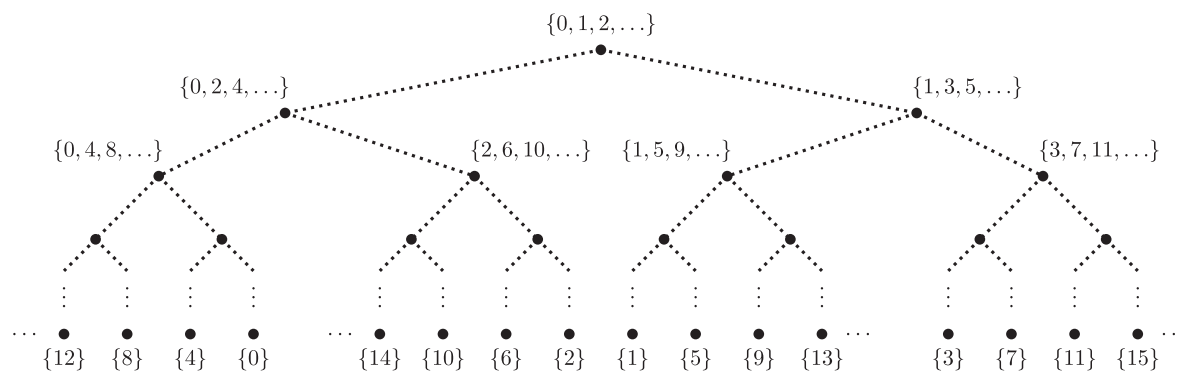

Fig. 4 A fragment of model $\mathcal{M}_{3}$

What logical relationships hold amongst superatomism, strong atomism, and standard atomism in CEM? I will reason model-theoretically, and restrict my attention to those models of CEM in which at least standard atomism is true. They include models with finitely many atoms. In every such model, every subset of its domain has an $\unlhd$-minimal element, i.e., parthood is well-founded in such models. As a result, every proper parthood chain terminates (every chain — not just every maximal chain). Were this the only type of model of CEM in which standard atomism is true, then superatomism, strong atomism, and standard atomism would each be equivalent to one another in CEM. But there are also models of CEM in which standard atomism is true with infinitely many atoms, such as $\mathcal{M}_{3}$. However, as I will argue next, whether such a model has denumerably or uncountably many atoms, it will contain maximal non-terminating chains.

I have already noted that models with denumerably many atoms, like $\mathcal{M}_{3}$, exhibit only a single structure: that imposed by the subset relation on the set of all non-empty subsets of $\{0,1, \ldots\}$. And while $\mathcal{M}_{3}$ includes maximal chains that are well-founded, such as that ending in $\ldots,\{0,1,2\},\{0,1\},\{0\}$, it also includes maximal chains with initial segments that do not terminate, where

Initial Segments. $\Gamma$ form an initial segment of $\Delta=_{d f}$ (i) $\Delta$ form a chain, (ii) there is an $x \preceq \Gamma$, (iii) $\Gamma \preceq \Delta$, and (iv) every $y$ among $\Delta$ but not among $\Gamma$ is a proper part of every $x$ among $\Gamma$.

(Each proper initial segment of a chain is the complement of a final segment of it.) An example of a maximal chain in $\mathcal{M}_{3}$ with an initial segment that does not terminate is that formed by $\{0,1,2, \ldots\},\{0,2,4, \ldots\},\{0,4,8, \ldots\}, \ldots,\{0\}$ (see the left end of Fig. 4), whose initial segment $\{0,1,2, \ldots\},\{0,2,4, \ldots\},\{0,4,8, \ldots\}, \ldots$ does not terminate. In addition, however, $\mathcal{M}_{3}$ includes maximal non-terminating chains as well. At least one fragment of $\mathcal{M}_{3}$ is isomorphic to $\mathcal{M}_{2}$, e.g., the one in which $b_{n}=\{n\}$ and $a_{n}=\{n, n+1, n+2, \ldots\}$ for each $n \in\{0,1, \ldots\}$. So the nonterminating chain formed by $\{0,1,2, \ldots\},\{1,2,3, \ldots\},\{2,3,4, \ldots\}, \ldots$ in $\mathcal{M}_{3}$ is maximal. For it to fail to be maximal, there would have to be a member of $\mathcal{D}_{3}$ that is a proper part of every member of the chain. By the definition of $\mathcal{M}_{3}$, this would mean that there would have to be a member of $\mathcal{D}_{3}$ that is a subset of every member of the chain. But there is no such member of $\mathcal{D}_{3}$. 
It would seem that other models of CEM with infinite numbers of atoms uncountably many - in which standard atomism is true also include maximal non-terminating chains. There will be at least one line of decomposition from any object composed of some atoms A, no matter their number, a final segment of which has the same structure as $\mathcal{M}_{2}$. It will be one of the lines in which a single one of its atomic parts is "peeled off" at each step. For the same reason as in the denumerable case, none of the atoms among A can be a proper part of everything in the nonterminating chain involved in that line of decomposition. There would have to be a member of the domain that is a subset of every member of the chain. But there will be no such member. Each member that is a subset of some member of the chain will have already been peeled off at the $\alpha$ th step for some ordinal $\alpha$, which can be infinite when there are uncountably many atoms. What this discussion shows is that every model of CEM in which standard atomism is true either (i) is well-founded (it has finitely many atoms) or (ii) is such that both superatomism and strong atomism are false (it has infinitely many atoms).

If my reasoning is sound, then several things follow. First, it follows that

\section{Proposition 9 Superatomism and strong atomism are equivalent in CEM.}

Given that (i) or (ii), every model of CEM in which standard atomism is true is such that superatomism and strong atomism are both true or both false. So there is no model in which one is true and the other is false. (The models I have not considered in which standard atomism is false - are models in which both superatomism and strong atomism are false as well. I show below that each of superatomism and strong atomism implies standard atomism in CEM.) Second, it follows that

Proposition 10 There is a model of CEM in which standard atomism is true but each of superatomism and strong atomism is false.

$\mathcal{M}_{3}$ is such a model. Everything in it is composed of atoms on each of the three standard definitions of fusion. Everything in the domain of $\mathcal{M}_{3}$ has a singleton subset, and so, by the characterization of $\mathcal{M}_{3}$, it has an atomic part. By Proposition 7 (that atomicity implies standard atomism), everything in the domain of $\mathcal{M}_{3}$ is composed of atoms. (Remember that Varzi's proof does not presuppose the falsity of CEM given any of the three definitions of fusion.) But $\mathcal{M}_{3}$ contains maximal non-terminating chains.

So if

Proposition 11 Each of superatomism and strong atomism implies standard atomism in $\boldsymbol{C E M}$.

then

Proposition 12 Each of superatomism and strong atomism is strictly stronger than standard atomism in CEM. 
Since superatomism and strong atomism are equivalent in CEM, it suffices to establish Proposition 11 that just one implies standard atomism. The proofs of Propositions 6 and 7 suffice to establish Proposition 11, given the Hausdorff maximal principle. But Uzquiano [21, 237] proves that superatomism implies atomicity without relying on this principle. Though he only shows this on the definition fusion 1, as I noted above, the three definitions of fusion are equivalent in CEM. So Uzquiano's proof, together with Varzi's proof of Proposition 7 (which does not presuppose the falisty of CEM), suffices to establish Proposition 11 without qualification.

To summarize: in this section, I have shown that superatomism and strong atomism are equivalent in CEM, and that each is strictly stronger than standard atomism in CEM. So in every mereology as weak as $\mathbf{M}$ and as strong as CEM, standard atomism is strictly weaker than, and so not equivalent to, either superatomism or strong atomism (though I relied on the Hausdorff maximal principle to show that strong atomism implies standard atomism in EM and strictly weaker M-mereologies). And while superatomism and strong atomism are equivalent in CEM, the former is strictly stronger than the latter in each of the M-mereologies strictly weaker than CEM. I turn now to philosophical matters related to these results.

\section{Which Principle is More Defensible, and Which, if any, Captures Atomism?}

I have shown two things. First, superatomism is strictly stronger than strong atomism, and (given the Hausdorff maximal principle) strong atomism is strictly stronger than standard atomism in EM and strictly weaker $\mathbf{M}$-mereologies. Second, superatomism and strong atomism are equivalent and each is strictly stronger than standard atomism in CEM. The various non-equivalencies between these principles (which, by themselves, do not rely on the Hausdorff maximal principle) show that atomism cannot be characterized by all three of them. No one has suggested that superatomism is equivalent to either of the other two. It has been presented by Cotnoir [3] and discussed by Uzquiano [21] as an alternative to atomism. But, as I noted in Section 3 above, various things that Shiver [17] says suggest that he may take strong atomism and standard atomism to be equivalent. We now know this is not the case in any of the mereologies I have discussed, which are the most common, and which are the systems Shiver considers.

Two less technical, more philosophical questions now present themselves.

(Q1) Which of superatomism, strong atomism, and standard atomism is the most defensible addition to one's mereology?

(Q2) Which of the them, if any, should be regarded as the best formulation of the atomistic thesis?

In what follows, I argue that standard atomism is a more defensible addition to one's mereology than either strong atomism or superatomism. That is, if one is to add one of them to one's mereology, one has more reason to add standard atomism than either of the other two. I also argue that standard atomism should be regarded as the best formulation of the atomistic thesis. (Q2) looks on its face tricker to answer 
than (Q1). It seems difficult for the proponent of one principle to avoid begging the question against her opponents. At first glance, at least, there does not appear to be any content to the idea of atomism, unless it is cashed out in one of these three ways. ${ }^{14}$ In what follows, however, I will identify some independent grips on the notion that one can utilize. On those bases, I believe that (Q2) can be answered. Doing so is easier once I have argued that (Q1) should be answered in the way I have suggested - that standard atomism is more defensible than its competitors. So I will proceed in that order. But again it will be helpful to break the discussion into two parts, one in which I assume EM or any strictly weaker M-mereology and one in which I assume CEM. Since my discussion of CEM is shorter, I begin with it.

A compelling argument that standard atomism is more defensible than its stronger alternative (superatomism/strong atomism) in the context of CEM is that, as Uzquiano [21] notes, were one to adopt the stronger option, one would rule out models that have infinitely many objects, such as $\mathcal{M}_{3} .{ }^{15}$ I see no reason for the proponent of CEM to want to rule out such models. Why couldn't there be infinitely many objects? And, concerning (Q2), it would be rather strange if one's characterization of atomism ruled out models with infinitely many objects, as the question of whether atomism is true - however that claim is ultimately cashed out - seems like it should not make determinations about the number of atoms there could be.

Another argument that standard atomism is more defensible than superatomism/strong atomism in the context of CEM is that, as Cotnoir [3, 69] and n. 5 points out, superatomism is incompatible with a "pointy" view of simples, where an object is pointy iff it exactly occupies a single point of space (see [13, 216]). It is incompatible with extended objects whose simple parts are pointy. The mereological structure of such objects will contain non-terminating chains, some maximal, some non-maximal, for the same reason that $\mathcal{M}_{3}$ does (see above). The equivalence between superatomism and strong atomism in CEM means of course that strong atomism also has this limitation. And again, it would be strange if atomism made determinations about whether such objects could exist. Thus not only should the proponent of CEM regard standard atomism as more defensible than its alternative, she should regard standard atomism as best capturing the atomistic thesis.

As I indicated, there are considerations in favor of thinking that the proponent of EM or a strictly weaker M-mereology should regard standard atomism as more defensible than its stronger alternatives as well. Before discussing them, however, I will consider and reply to what I take to be three of the most promising arguments in favor of adopting a stronger alternative to standard atomism. Each of these arguments

\footnotetext{
${ }^{14}$ It is tempting to say that, when it comes to the question of which of the three principles best captures the atomistic thesis, the burden of proof lies on the proponent of the claim that the atomistic thesis is best captured by superatomism or strong atomism, as standard atomism appears to be the most straightforward way of capturing the atomistic thesis. But the proponent of one of the stronger principles can reply by simply saying that this is a mistake.

${ }^{15}$ Uzquiano [21, 237] provides a proof of this claim. But the point can be appreciated by remembering from that the only superatomistic models of CEM are ones with finitely many atoms, and observing that, given CEM, the only atomistic models which feature an infinite number of objects are ones which feature an infinite number of atoms.
} 
is one against the possibility of non-terminating chains in general, and as such is an argument for superatomism rather than just either strong atomism or standard atomism. First, an object which contains a non-terminating proper parthood chain cannot be built from its basic constituents (either by intelligent agency or by nature), as the progression of steps required to build it, bottom to top, is not well-ordered. This is the constructibility argument. Second, it may be problematic that not everything in such a chain can be decomposed into its ultimate constituents. This is the decomposition argument (see [24, 2894-95]). Third, such a chain results in violations of the well-foundedness of grounding when one supposes (as is plausible) that composite objects metaphysically depend on their proper parts (see [3] and [24, 2895]). This is the foundationalist argument.

Regarding the constructibility argument, many composite objects presumably exist which were not built from their basic constituents, such as space or the universe itself. Regarding the decomposition argument, I grant that not everything in a model which contains a maximal non-terminating proper parthood chain, like $\mathcal{M}_{1}$ or $\mathcal{M}_{2}$, can be decomposed into its ultimate constituents in a finite number of steps. But I do not see why the question of whether or not something can be decomposed into its ultimate constituents simpliciter depends on that of whether it can be decomposed in a finite number of steps. Moreover, I do not see why the fact that something cannot be decomposed into its ultimate constituents should prevent that object from existing. Why should the fact that non-eternal beings incapable of supertasks cannot deconstruct an object (mentally or physically) prevent that object from existing? ${ }^{16}$

Before replying to the foundationalist argument, let me explicate it in more detail. Recall that the basic concern is that the existence of a non-terminating proper parthood chain will result in violations of the well-foundedness of grounding when one presupposes that composite objects depend on their proper parts. The precise principle follows.

(PPG) For any $x$ and $y$, if $y$ is a proper part of $x$, then $x$ is partially grounded by $y$.

\footnotetext{
${ }^{16}$ Uzquiano makes a point concerning decomposition in favor of the claim that standard atomism best captures the atomistic thesis.
}

Atomism makes sure that every fusion is composed of atoms, but it [was] never meant to place further restrictions on the range of available patterns of decomposition for a given fusion. [21, 234]

Strong atomism does so restrict the available patterns of decomposition for fusions. For example, it prevents a thing from being composed of infinitely many atoms if that thing can only be decomposed in the way that $\mathcal{M}_{2}$ can be decomposed: in an infinite number of steps, where there is no last step in that decomposition. This is the nature of a maximal non-terminating chain. According to Uzquiano, atomism is a thesis about composition, not about decomposition. If he is right, then whether or not it is possible to decompose something in a certain way is irrelevant to whether that thing is atomistic. Varzi [24, 2895] expresses a similar sentiment. Remarks of this sort suggest that any attempt to ensure our atomism axiom proscribes maximal non-terminating chains would rest on a conflation of these two issues. While I am sympathetic to this view, I am also cognizant of the concern I raised above; it seems that the proponent of the view that strong atomism is the best way to capture atomism will simply deny that atomism is not a thesis about decomposition. So I don't think that Uzquiano's point constitutes as decisive a reason to think that standard atomism is the best way to characterize atomism as was available in the context of CEM. 
Grounding is metaphysical dependence. For $x$ to be grounded by $\Gamma$ is for $x$ to metaphysically depend on $\Gamma$. But there is a distinction between full grounding and partial grounding. One way to think of $x$ being fully grounded by $\Gamma$ is to suppose that, in addition to $x$ metaphysically depending on $\Gamma$, the existence of $\Gamma$ metaphysically necessitates the existence of $x .^{17}$ The existence of some partial grounds of something need not metaphysically necessitate it. So, for example, the fact that both $p$ and $q$ is fully grounded by $p$ together with $q$. Neither $p$ nor $q$ may fully ground the fact that both $p$ and $q$, though the latter will be partially grounded by each taken individually. Partial grounding is typically defined in terms of full grounding as follows.

Partial Grounding. $x$ is partially grounded by $\Gamma={ }_{d f}$ there are $\Delta$ such that (i) $\Gamma \preceq \Delta$ and (ii) $x$ is fully grounded by $\Delta .{ }^{18}$

A model with the structure of $\mathcal{M}_{1}$ or $\mathcal{M}_{2}$ would be considered problematic because (PPG) would guarantee that a non-terminating proper parthood chain would result in a non-terminating grounding chain, the latter of which is defined in a way that is analogous to the way the former is defined.

Grounding Chains. $\Gamma$ form grounding chain $=_{d f}$ (i) there are $x, y \preceq \Gamma$ such that $x$ is partially grounded by $y$ and (ii) for every $x, y \preceq \Gamma$, either $x$ is partially grounded by $y, y$ is partially grounded by $x$, or $x=y$.

(Downwardly) Terminating Grounding Chains. $\Gamma$ form a (downwardly) nonterminating grounding chain $=_{d f}$ (i) $\Gamma$ form a grounding chain and (ii) there is a $y \preceq \Gamma$ that partially grounds every other $x \preceq \Gamma$.

The existence of such a chain conflicts with the claim that grounding is well-founded which, like superatomism, requires that every chain terminates.

It is certainly true that, given (PPG), non-terminating proper parthood chains result in conflicts with the claim that grounding is well-founded, where that claim is understood as the claim that grounding is a well-founded relation in the mathematical sense, i.e., that every set $S$ of the domain of grounding has a ground-minimal element (an element which isn't grounded by anything in S), and implies that every grounding chain terminates. However, the operative premise in the argument seems to be that metaphysical foundationalism is true, where metaphysical foundationalism is a certain view about how reality is structured by grounding. If metaphysical foundationalism best captured as the claim that grounding is well-founded in the mathematical sense, then the argument is sound. But as Dixon [5] argues and Rabin and Rabern [16, 363-64] suggest, the most plausible way to characterize metaphysical foundationalism is as follows.

\footnotetext{
${ }^{17}$ Not everyone endorses the claim that full grounds necessitate what they ground. See, for example, [19]. But supposing this provides a useful way to get an intuitive handle on the notion of full grounding, especially as it contrasts with that of partial grounding. For a general introduction to the notion of grounding, see [1].

${ }^{18}$ This definition has its skeptics. Leuenberger [10], for example, argues that something can be partially grounded by something that is not among any full grounds of it. As it happens, however, the foundationalist argument depends only on the less controversial right-to-left direction: that everything among a thing's full grounds is a partial ground of it.
} 
Full Foundations. For every non-fundamental entity $x$, there are some fundamental entities that fully ground $x$,

where a fundamental entity is one which has no grounds and a non-fundamental entity is one that is not fundamental. And, as Dixon [5, Section 7], Litland [12], and Rabin and Rabern $[16,366]$ note, full foundations is compatible with the existence of non-terminating grounding chains - both non-maximal and maximal, where

(Downwardly) Maximal Grounding Chains. $\Gamma$ form a (downwardly) maximal grounding chain $=_{d f}$ (i) $\Gamma$ form a grounding chain and (ii) there is no $y$ that partially grounds every $x \preceq \Gamma$.

(PPG) would presumably be adopted as a result of adopting a stronger but no less plausible principle connecting composition to full grounding.

(CFG) For any $x$ and $\Gamma$, if $x$ is composed of $\Gamma$, then $x$ is fully grounded by $\Gamma$.

Neither $\mathcal{M}_{1}$ nor $\mathcal{M}_{2}$, nor any model with either structure, results in a violation of full foundations once (CFG) is applied (which one would no doubt apply along with (PPG)), as long as, in $\mathcal{M}_{1}, a_{n}$ is composed of $b, c_{n}, c_{n+1}, \ldots$ for every $n \in\{0,1, \ldots\}$ by each definition of fusion (which the reader can confirm is the case), and, in $\mathcal{M}_{2}$, $a_{n}$ is composed of $b_{n}, b_{n+1}, \ldots$ for every $n \in\{0,1, \ldots\}$ by each definition of fusion (which Varzi [24, 2894], fn. 5 proves). I'll focus my attention on $\mathcal{M}_{2}$. Once (CFG) is applied, every non-fundamental entity $a_{n}$ in it (i.e., every composite entity) is fully grounded by some fundamental entities $b_{n}, b_{n+1}, \ldots$ (i.e., by some atomic entities) for every $n \in\{0,1, \ldots\}$. It appears, then, that the foundationalist argument will not support the claim that all non-terminating proper parthood chains should be prohibited either, at least if Dixon and Rabin and Rabern are right about how best to formulate metaphysical foundationalism. Nor will it even support strong atomism. ${ }^{19}$

\footnotetext{
${ }^{19}$ Note that full foundations is weaker than the claim that every grounding chain terminates (see [5, Section 4] and [16, 362-67]). And it is weak enough to allow one to avoid Cotnoir's [3] conclusion that superatomism is true, which he shows follows from (i) the claim that parthood is a partial order, (ii) (PPG), and (iii) the well-foundedness of grounding, by allowing one to reject (iii). Moreover, as Dixon [5, Section 67] and Rabin and Rabern [16, Thms. 9 and 10] show, full foundations is logically independent from the claim that every maximal grounding chain terminates, and thus represents a way of weakening the thesis that grounding is well-founded that is different than that considered by Cotnoir [3, 70], and which, crucially, seems adequate to its purpose. As Dixon $[5,454-55]$ notes, the claim that every maximal grounding chain terminates is inadequate as a characterization of metaphysical foundationalism since it allows for models which, intuitively, are not foundationalist. Interestingly, although the claim that every maximal grounding chain terminates is logically independent from full foundations, strong atomism, along with (CFG), implies full foundations if it is assumed that every non-fundamental entity is composite (and so every atom is fundamental). (While this may be implausible in general, it is benign in this context because I am here concerned only with the implications of parthood claims on the domain of grounding.)
}

Proof Consider an arbitrary non-fundamental entity $a$. Since $a$ is non-fundamental, $a$ is composite. By Propositions 6 and 7, strong atomism implies that everything is composed of atoms (on all three of the standard definitions of fusion). So $a$ is composed of some atoms. By (CFG), $a$ is fully grounded by atoms. Since every atom is fundamental, $a$ is fully grounded by some fundamental entities. Since $a$ is arbitrary, every non-fundamental entity is fully grounded by fundamental entities. 
To the extent that my replies are convincing, they prevent the three arguments I have just considered from establishing the metaphysical impossibility of nonterminating proper parthood chains, maximal or otherwise. I will now discuss a consideration for the metaphysical possibility of maximal non-terminating proper parthood chains, and thus in favor of the idea that standard atomism is preferable to either strong atomism or superatomism. This will come in the form of an apparently metaphysically possible object that has a mereological structure which contains a substructure isomorphic to the structure of $\mathcal{M}_{2}$, and so includes at least one such chain. This will constitute my case that standard atomism is more defensible than strong atomism in EM and strictly weaker mereologies. ${ }^{20}$ But I hope that it will also have another effect. In particular, I hope that it will remove some of the perplexity to which Uzquiano [21, 233] alludes about models like $\mathcal{M}_{2}$. A realistic interpretation of such models should help convince us that maximal non-terminating proper parthood chains are not as offensive as we might be inclined to think when we consider them only in the abstract.

Recall that we are now working within the context of non-classical EM. So not every collection of objects in the domain of a model will necessarily have a fusion in it. CEM is controversial in this respect anyway, since it allows for the possibility of composite objects which intuitively don't exist. Many think that, in order for there to be something that is composed by some things, those things must meet a condition stronger than mere existence, e.g., they must be attached to one another in some sense (see [22, chs. 2-7]). So, while many would presumably assent to the claim that Great Britain and Northern Ireland compose an object (the United Kingdom); England, Wales, and Scotland compose an object (Great Britain); and England, Wales, Scotland, and Northern Ireland compose an object (the United Kingdom), many would presumably deny that, for any $\Gamma$ among England, Wales, Scotland, and Northern Ireland, $\Gamma$ compose an object. The only composition claims that hold of any of the UK, Great Britain, England, Wales, Scotland, and Northern Ireland appear to be those just mentioned, depicted by the boxes in Fig. 5. An object with the mereological structure represented by $\mathcal{M}_{2}$ is possible if we think there can be an object akin to the United Kingdom insofar as it can only be decomposed in certain ways, but which differs only in that that decomposition would take an infinite number of steps.

Such objects appear to be metaphysically possible, at least if one is not presuming the truth of CEM. Suppose, for example, that there is a staircase made of indivisible slabs, which are mereological atoms, each with a thickness of one foot, that starts at sea level on flat Earth and runs downward forever. And suppose that, for whatever reason, only singular and infinite sequential collections of these slabs can compose objects. (Numerical restrictions on the conditions under which some things of a certain type compose a larger object are not unprecedented outside of a classical setting. Distinct quarks, after all, can, in such a setting, naturally be taken to compose objects

\footnotetext{
${ }^{20}$ One might argue that the fact that, of the three principles, only standard atomism can be specified in singular first-order logic, which is the language underlying most developments of mereology, is a point in its favor. See fn. 7. Because I do not find the use of plural first-order logic objectionable, I will not pursue this argument here.
} 


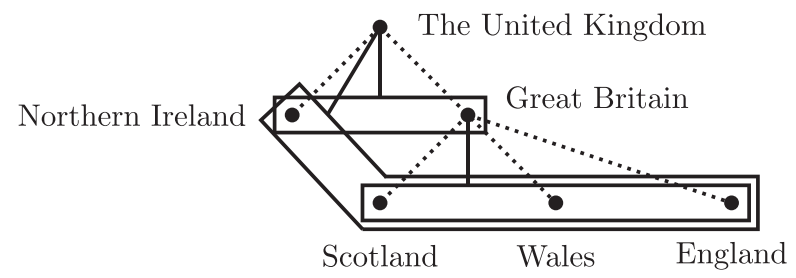

Fig. 5 Large geographical entities in the British Isles. A solid line connecting a node $x$ to a solid box enclosing nodes $y_{1}, y_{2}, \ldots$ indicates that $x$ is composed of $y_{1}, y_{2}, \ldots$

only in threes.) Under these assumptions, the staircase can only be decomposed in one way: by removing slabs one at a time from the top downwards. Its immediate proper parts are the top slab and the staircase that starts one foot below sea level. The immediate proper parts of that staircase, in turn, are the penultimate slab and the staircase that starts two feet below sea level. And so on. This object would have the mereological structure of $\mathcal{M}_{2}$. And, while it would be impossible for any craftsperson $I$ know to construct such a staircase, it seems metaphysically possible. ${ }^{21}$

So adopting a principle stronger than standard atomism seems neither wellmotivated nor without costs, in the sense that the best arguments for doing so are suspect, and doing so forces one to exclude the possibility of seemingly metaphysically possible objects. But there are reasons, even in the case of EM and strictly weaker mereologies, to think that not only is standard atomism more defensible than its rivals, it best captures the atomistic thesis. I present two such reasons. The first is methodological. Standard atomism is strictly the weakest of the three principles no matter what mereology one is working with. The other two change their strength relative to one another when one moves from a mereology strictly weaker than CEM to CEM. Thus standard atomism is unique in that it can act as a baseline, regardless of one's preferred mereology, below which there are objects which are definitely offensive to the atomistically inclined, and above which there might be some debate.

My second reason for thinking that standard atomism best captures atomism is that the whole point of being an atomist, it seems to me, is to exclude the possibility of gunk, where

Gunk. $x$ is gunk $=_{d f}$ every part of $x$ has a proper part.

Atomism, is, after all, traditionally represented as the alternative to the possibility of gunk (see, for example, [18, 41-42]). And it turns out that the claim that there is no gunk is equivalent to the claim that everything is composed of atoms (standard atomism) in all of the mereologies I have considered.

Proposition 13 The claim that there is no gunk is equivalent to standard atomism in CEM and strictly weaker $\boldsymbol{M}$-mereologies.

\footnotetext{
${ }^{21}$ Other similar examples include an infinite set of Russian dolls, the outermost one of finite size, and every other half the width of the one encapsulating it, and an onion of finite size with an infinite number of layers, each inner layer half the thickness of the one encapsulating it. Thanks to Martin Pleitz and Martin Glazier (personal communications) for respectively suggesting these examples.
} 
I prove this by first proving that atomicity (the claim that everything has an atomic part) is equivalent to standard atomism. Second, I prove that the claim that there is no gunk is equivalent to atomicity. Thanks to Varzi, we know that atomicity implies standard atomism in every mereology I have considered and given any of the three standard definitions of fusion. ${ }^{22}$ And standard atomism implies atomicity in every such mereology and on every such definition as well. If everything is composed of some atoms, then everything has an atomic part. The only case in which this is not immediately obvious given the definitions of composition and fusion is in the case of fusion 2.

Proof Suppose that everything is composed of atoms and consider an arbitrary $a$. Either $a$ is atomic or composite. If $a$ is atomic, then it has itself as an atomic part. If $a$ is composite, then $a$ is composed of atoms B. It follows that $a$ fuses B, and so $a$ overlaps all and only those things which overlap at least one $y \preceq$ B. Consider an arbitrary $b \preceq$ B. $b$ overlaps itself, and so overlaps at least one $y \preceq$ B. Since $b$ is atomic, it has no proper parts. So the only way it can overlap $a$ is for it to be (a proper) part of $a$. Since B exist, so does $b$, and so $a$ has an atomic part.

Note that this proof relies on no special assumptions about parthood, and so establishes the claim in all of the mereologies of interest. As to the second equivalence, it is obvious that atomicity implies that there is no gunk, since, if there were, something would fail to have any atomic parts. ${ }^{23}$ And it is easy to prove that the claim that there is no gunk implies atomicity.

Proof Suppose that there is no gunk, and consider an arbitrary $a$. Then some part $b$ of $a$ has no proper parts. That is, $b$ is an atom. Since $a$ is arbitrary, everything has an atomic part.

These last two implications depend only on the definitions of gunk and atomicness. Proposition 13 follows by the transitivity of logical equivalence. The significance of this result is that if the whole point of being an atomist is to rule out gunk, then standard atomism does the job. If one wants to adopt a stronger principle, that is one's right. But one will be adopting a system that is stronger than an otherwise identical atomistic system, and one will have to find another reason to justify it. But, as I discussed in this section, compelling motivation for that move is yet to be found. And in making that move, one will have to rule out seemingly metaphysically possible scenarios, like the existence of infinitely many objects, the existence of objects composed of pointy simples, and the existence of objects like the infinitely descending staircase.

\footnotetext{
${ }^{22}$ With the exception that it holds in the case of fusion 3 only in mereologies at least as strong as EM. See the discussion of Proposition 7 in Section.

${ }^{23}$ Tsai and Varzi [20] construct a model of EM in which atomicity is true but in which, they allege, some elements of its domain have parts which "can be divided indefinitely into smaller and smaller proper parts" (Ibid.: 232). However this claim is understood, as Uzquiano notes [21, n. 8], it cannot be understood as the claim that every composite object in the model has gunky parts, and so does not count as a counterexample to the claim that atomicity implies that there is no gunk.
} 


\section{Concluding Remarks}

I showed that superatomism is strictly stronger than strong atomism, which (given the Hausdorff maximal principle) is strictly stronger than standard atomism in EM and strictly weaker M-mereologies. I also showed that each of superatomism and strong atomism, while equivalent to the other in CEM, is strictly stronger than atomism in CEM. I then argued that standard atomism is more defensible than either of its stronger counterparts no matter which of these mereologies one adopts, and argued further that it, and neither of its stronger counterparts, best captures the atomistic thesis. As a result, we should take the possibility of non-terminating proper parthood chains (maximal or not) seriously. And we should not conclude that a model is not atomistic simply because it contains a maximal non-terminating proper parthood chain.

Acknowledgements Open Access funding provided by Projekt DEAL. I am grateful to several anonymous referees, and to an audience at the Forschungskolloquium at Universität Hamburg for helpful comments on an ancestor of this paper. Thanks also to Cody Gilmore, Michael Glanzberg, Paul Hovda, and Martin Pleitz for helpful discussions on specific issues related to this paper (noted above), and to an anonymous referee for extremely helpful and extensive comments on it.

\section{Compliance with Ethical Standards}

Conflict of interests The authors declare that they have no conflict of interest.

Open Access This article is licensed under a Creative Commons Attribution 4.0 International License, which permits use, sharing, adaptation, distribution and reproduction in any medium or format, as long as you give appropriate credit to the original author(s) and the source, provide a link to the Creative Commons licence, and indicate if changes were made. The images or other third party material in this article are included in the article's Creative Commons licence, unless indicated otherwise in a credit line to the material. If material is not included in the article's Creative Commons licence and your intended use is not permitted by statutory regulation or exceeds the permitted use, you will need to obtain permission directly from the copyright holder. To view a copy of this licence, visit http://creativecommonshorg/licenses/by/4.0/.

\section{Appendix}

Proof of Proposition 6 Suppose that every maximal proper parthood chain terminates and consider an arbitrary $a$. I set out to show that $a$ has an atomic part. Suppose for reductio that $a$ does not have an atomic part and define $\mathrm{Z}$ so that, for any $x, x \preceq \mathrm{Z}$ iff $x \triangleleft a$. Since every atom has itself as an atomic part, $a$ must be composite; hence it has a proper part, and so $\mathrm{Z}$ exist.

(1) First I show that, for any $x \preceq Z$, there is a $y \preceq Z$ such that $y \triangleleft x$. Consider an arbitrary $b \preceq$ Z. $b$ isn't atomic, since, if it were, $a$ would have an atomic part, contrary to the initial reductio assumption. Hence $b$ is composite, and so has a proper part $c$. By the transitivity of proper parthood, $c \triangleleft a$. But then by the characterization of $\mathrm{Z}, c \preceq \mathrm{Z}$. So there is a $y \preceq \mathrm{Z}$ such that $y \triangleleft b$.

(2) Next I show that there is a maximal chain among $Z$. Consider again an arbitrary $b \preceq \mathrm{Z}$. From the previous result, $b$ has a proper part $c \preceq \mathrm{Z}$. So there is a chain 
formed by $b, c=\mathrm{Z}_{\mathrm{C}}$, where $\mathrm{Z}_{\mathrm{C}} \preceq \mathrm{Z}$. Since $\triangleleft$ partially orders its domain, the Hausdorff maximal principle guarantees that there is a maximal chain formed by some $\mathrm{M}$ such that $\mathrm{Z}_{\mathrm{C}} \preceq \mathrm{M}$.

(3) I now set out to show that the chain formed by $\mathrm{M}$ has as a final segment a chain formed by objects among $\mathrm{Z}$ that is both maximal and does not terminate. Define $\mathrm{N}$ so that, for any $x, x \preceq \mathrm{N}$ iff $x=b$ or both $x \preceq \mathrm{M}$ and $x \triangleleft b$. Since $b$ exists, $\mathrm{N}$ exist.

(a) I now show that $\mathrm{N}$ form a final segment of the chain formed by $\mathrm{M}$.

(i) I first show that $\mathrm{N} \preceq \mathrm{M}$. Consider an arbitrary $d \preceq \mathrm{N}$. Whether $d=b$ or $d \triangleleft b$, by the characterization of $\mathrm{N}, d \preceq \mathrm{M}$. Since $d$ is arbitrary, for every $x \preceq \mathrm{N}, x \preceq \mathrm{M}$.

(ii) I now show that every $x \preceq \mathrm{N}$ is a proper part of every $y \preceq \mathrm{M}$ such that $y \npreceq \mathrm{N}$. Consider arbitrary $d \preceq \mathrm{N}$ and $e \preceq \mathrm{M}$ where $e \npreceq \mathrm{N}$. (If there is no such $e$, the goal follows trivially.) By the characterization of $\mathrm{N}$, either $d=b$ or both $d \preceq \mathrm{M}$ and $d \triangleleft b$. Since $d, e \preceq \mathrm{M}$ and $\mathrm{M}$ form a chain, either $d \triangleleft e, e \triangleleft d$, or $d=e$. If $e \triangleleft d$ then, if $d=b$, it immediately follows that $e \triangleleft b$. And if $d \triangleleft b$, the transitivity of proper parthood guarantees that $e \triangleleft b$. And since $e \preceq \mathrm{M}$, the characterization of $\mathrm{N}$ guarantees that $e \preceq \mathrm{N}$, contradicting the assumption that $e \npreceq \mathrm{N}$. If $e=d$, it also follows that $e \preceq \mathrm{N}$, since it was assumed that $d \preceq \mathrm{N}$, again contradicting the assumption that $e \npreceq \mathrm{N}$. So $d \triangleleft e$.

(b) I now show that $\mathrm{N} \preceq \mathrm{Z}$. Consider an arbitrary $d \preceq \mathrm{N}$. By the characterization of $\mathrm{N}, d=b$ or $d \triangleleft b$. If $d=b$, then, since $b \preceq \mathrm{Z}$, the characterization of Z guarantees that $b \triangleleft a$, and so $d \triangleleft a$. If $d \triangleleft b$, then, since, for the same reason, $b \triangleleft a$, the transitivity of proper parthood guarantees that $d \triangleleft a$. Either way, $d \triangleleft a$. So by the characterization of Z, $d \preceq \mathrm{Z}$. Since $d$ is arbitrary, for every $x \preceq \mathrm{N}, x \preceq \mathrm{Z}$.

(c) I now show that $\mathrm{N}$ form a chain.

(i) I first show that there are $x, y \preceq \mathrm{N}$ such that $x \triangleleft y$. By the characterization of $\mathrm{N}, b, c \preceq \mathrm{N}$. It is also known that $c \triangleleft b$ (see step (2)).

(ii) I now show that, for any $x, y \preceq \mathrm{N}$, either $x \triangleleft y, y \triangleleft x$, or $x=y$. Consider arbitrary $d, e \preceq \mathrm{N}$. Since, by result (3)(a)(i) above, $\mathrm{N} \preceq \mathrm{M}$, the transitivity of inclusion guarantees that $d, e \preceq \mathrm{M}$. Since $\mathrm{M}$ is a chain, either $d \triangleleft e, e \triangleleft d$, or $d=e$.

(d) I show next that the chain formed by $\mathrm{N}$ is maximal. Suppose for reductio that it is not. Then every $x \preceq \mathrm{N}$ shares a proper part $d$. (Remember that the definition of maximal chains presupposes the transitivity of proper parthood.) Consider an arbitrary $e \preceq \mathrm{N}$. Then $d \triangleleft e$. Now consider an arbitrary $f \preceq \mathrm{M}$. Since M form a chain and, by (3)(a)(i), $e \preceq$ M, either (i) $e \triangleleft f$, (ii) $f \triangleleft e$, or (iii) $e=f$.

(i) Suppose $e \triangleleft f$. The transitivity of proper parthood guarantees that $d \triangleleft f$. 
(ii) Suppose $f \triangleleft e$. Since $e \preceq \mathrm{N}$, the characterization of $\mathrm{N}$ guarantees that $e=b$ or $e \triangleleft b$. If $e=b$, it immediately follows that $f \triangleleft b$. And if $e \triangleleft b$, the transitivity of proper parthood guarantees that $f \triangleleft b$. Since $f \preceq \mathrm{M}$, the characterization of $\mathrm{N}$ guarantees that $f \preceq \mathrm{N}$. And since $d$ is a proper part of every $x \preceq \mathrm{N}, d \triangleleft f$.

(iii) Suppose $e=f$. Then since $d \triangleleft e, d \triangleleft f$.

Any way, $d \triangleleft f$. And since $f$ is arbitrary, $d$ is a proper part of every $x \preceq \mathrm{M}$. So some $y$ is a proper part of every $x \preceq$ M. It now follows that the chain formed by $\mathrm{M}$ is not maximal, contradicting the result of the above application of the Hausdorff maximal principle (see step (2)), which ensures that it is.

(e) I now show that the chain formed by $\mathrm{N}$ does not terminate. Suppose for reductio that it does. Then there is a $d \preceq \mathrm{N}$ such that, for every $x \preceq \mathrm{N}$, if $x \neq d$ then $d \triangleleft x$. Since, by result (3)(b), $\mathrm{N} \preceq \mathrm{Z}, d \preceq \mathrm{Z}$. Then, by result (1), $d$ has a proper part $e \preceq$ Z. Now consider an arbitrary $f \preceq \mathrm{N}$. Either $f=d$ or $f \neq d$.

(i) Suppose $f=d$. Then, since $e \triangleleft d, e \triangleleft f$.

(ii) Suppose $f \neq d$. Then, by the reductio assumption, $d \triangleleft f$. But since $e \triangleleft d$, the transitivity of proper parthood guarantees that $e \triangleleft f$.

Either way, $e \triangleleft f$. Since $f$ is arbitrary, every $x \preceq \mathrm{N}$ has $e$ as a proper part. But by result (3)(d), $\mathrm{N}$ is maximal, and so there is no $y$ that is a proper part of every $x \preceq \mathrm{N}$. Contradiction. So the chain formed by $\mathrm{N}$ does not terminate.

But since, by result (3)(d), the chain formed by $\mathrm{N}$ is maximal, the initial assumption guarantees that it terminates. Contradiction. Hence $a$ has an atomic part. And since $a$ is arbitrary, everything has an atomic part.

\section{References}

1. Bliss, R., \& Trogdon, K. (2014). Metaphysical grounding. In Zalta, E.N. (Ed.) Stanford encyclopedia of philosophy. Winter 2016 edn. https://plato.stanford.edu/archives/win2016/entries/grounding/.

2. Boolos, G. (1984). To be is to be a value of a variable (or to be some values of some variables). Journal of Philosophy, 81, 430-49. https://doi.org/10.2307/2026308.

3. Cotnoir, A.J. (2013). Beyond atomism. Thought, 2, 67-72. https://doi.org/10.1002/tht3.64.

4. Devlin, K. (1993). The Joy of Sets, 2nd edn. New York: Springer.

5. Dixon, T.S. (2016). What is the well-foundedness of grounding? Mind, 125, 439-68. https://doi.org/10.1093/mind/fzv112.

6. Frink, O. (1952). A proof of the maximal chain theorem. American Journal of Mathematics, 74, 676-78. https://doi.org/10.2307/2372270.

7. Hausdorff, F. (1962). Set Theory, 2nd edn. New York: Chelsea Publishing Company.

8. Hovda, P. (2009). What is classical mereology? Journal of Philosophical Logic, 38, 55-82. https://doi.org/10.1007/s10992-008-9092-4.

9. Jech, T.J. (2002). Set theory, 3rd edn. Berlin: Springer.

10. Leuenberger, S. (2019). The fundamental: Ungrounded or all-grounding? Philosophical Studies, 1-23. https://doi.org/10.1007/s11098-019-01332-x.

11. Linnebo, Ø. (2017). Plural quantification. In Zalta, E.N. (Ed.) Stanford encyclopedia of philosophy. Summer 2017 edn. https://plato.stanford.edu/archives/sum2017/entries/plural-quant/.

12. Litland, J.E. (2016). An infinitely descending chain of ground without a lower bound. Philosophical Studies, 173, 1361-69. https://doi.org/10.1007/s11098-015-0550-x.

13. Markosian, N. (1998). Simples. Australasian Journal of Philosophy, 76, 213-28. https://doi.org/10. 1080/00048409812348361. 
14. McDaniel, K. (2010). Parts and wholes. Philosophy Compass, 5, 412-25. https://doi.org/10.1111/j. 1747-9991.2009.00238.x.

15. Oliver, A., \& Smiley, T. (2013). Plural logic. Oxford: Oxford University Press. https://doi.org/10.1093/acprof:oso/9780199570423.001.0001.

16. Rabin, G.O., \& Rabern, B. (2016). Well-founding grounding grounding. Journal of Philosophical Logic, 45, 349-379. https://doi.org/10.1007/s10992-015-9376-4.

17. Shiver, A. (2015). How do you say 'everything is ultimately composed of atoms'? Philosophical Studies, 172, 607-14. https://doi.org/10.1007/s11098-014-0321-0.

18. Simons, P.M. (1987). Parts: a study in ontology. Oxford: Oxford University Press. https://doi.org/10. 1093/acprof:oso/9780199241460.001.0001.

19. Skiles, A. (2015). Against grounding necessitarianism. Erkenntnis, 80, 717-51. https://doi.org/10. 1007/s10670-014-9669-y.

20. Tsai, H., \& Varzi, A.C. (2016). Atoms, gunk, and the limits of 'composition'. Erkenntnis, 81, 231-35. https://doi.org/10.1007/s10670-015-9736-z.

21. Uzquiano, G. (2017). Atomism and composition. Thought, 6, 232-40. https://doi.org/10.1002/ tht 3.256 .

22. van Inwagen, P. (1990). Material beings. Ithaca: Cornell University Press. https://doi.org/10.7591/ 9781501713033.

23. Varzi, A.C. (2016). Mereology. In Zalta, E.N. (Ed.) Stanford encyclopedia of philosophy. Spring 2016 edn. http://plato.stanford.edu/archives/spr2016/entries/mereology/.

24. Varzi, A.C. (2017). On being ultimately composed of atoms. Philosophical Studies, 174, 2891-2900. https://doi.org/10.1007/s11098-016-0816-y.

Publisher's Note Springer Nature remains neutral with regard to jurisdictional claims in published maps and institutional affiliations. 